\title{
El papel del género en la toma de decisiones bajo incertidumbre*
}

\author{
Aurora García-Gallego \\ LEE y Dpto. de Economía, U. Jaume I y U. de Chipre \\ Nikolaos Georgantzís \\ GLOBE y Dpto. de Economía, Universidad de Granada \\ Ainhoa Jaramillo-Gutiérrez \\ LEE y Dpto. de Economía, Universitat Jaume I
}

\section{Resumen}

Estudiamos las diferencias de género en la toma de decisiones individuales bajo incertidumbre. Para elicitar las preferencias frente al riesgo de nuestros sujetos, utilizamos el test de loterías introducido por Sabater-Grande y Georgantzís (2002). Este test, además de permitir el estudio de los datos primarios sobre la elección del individuo bajo incertidumbre, nos permite medir la sensibilidad del sujeto a las primas de riesgo. Encontramos que las mujeres son más aversas al riesgo que los hombres. Además, las mujeres son menos sensibles que los hombres a las primas de riesgo.

Palabras clave: actitud frente al riesgo, diferencias de género, experimentos económicos.

Códigos JEL: C91, D81, J16

\section{Abstract}

We study gender differences in individual decision making under uncertainty. To elicit risk preferences, we use the lottery panel test introduced in Sabater-Grande and Georgantzís (2002). This test allows us not only to study the primary data of individual decision making under uncertainty, but also to analyze the sensitivity to risk premia of our subjects' risk preferences. We find that females are more risk averse than males. Moreover, females are less sensitive to risk premia than males.

Keywords: risk attitudes, gender differences, economic experiments.

JEL codes: C91, D81, J16

\section{Introducción}

La toma individual de decisiones bajo incertidumbre depende tanto del contexto como de la idiosincrasia y de las características demográficas de quien toma la decisión. De todas ellas, el género es la característica más sistemáticamente estudiada. Sin embargo, mientras los efectos de género en la aversión al riesgo han sido

* Agradecemos los comentarios de G. Panos, M. Paz Espinosa y M. José Gil-Moltó. Esta investigación ha recibido ayuda financiera de fondos europeos (proyecto EPICURUS, SERD-2002-00057), del Ministerio de Educación y Ciencia (proyecto SEJ2005-07544/ECON), de la Junta de Andalucía (proyecto PO7-SEJ-03155) y de Bancaja (proyecto P1 B22007-14). Traducción a cargo de Joan Monrás. 
ampliamente documentados, otros ámbitos de la toma individual de decisiones bajo incertidumbre han recibido mucha menos atención. De forma excepcional, Harbaugh y otros (2002) y Fehr-Duda y otros (2006) abordaron la cuestión de los pesos que reciben las probabilidades dependiendo del género. De hecho, en la última de estas publicaciones se indica que las diferencias de género son específicas al rango de probabilidades asociado a unas ciertas ganancias o pérdidas.

La ponderación de probabilidades parece ser un importante proceso en las elecciones individuales arriesgadas, e intentaremos remarcar las cuestiones que trataremos en este artículo de la forma más general posible. Tras décadas de investigación teórica, empírica y experimental en este campo, todavía son pocos los ámbitos en los que los economistas han adoptado un punto de vista unánime, compatible con las diferentes teorías y hechos aceptados. Posiblemente, el principio más ampliamente aceptado pueda resumirse de la siguiente manera: el ser humano prefiere proyectos más rentables siempre y cuando la incertidumbre asociada no sea demasiado alta, en cuyo caso preferirá alternativas menos rentables pero también menos arriesgadas.

En este marco general, estudiamos diferencias de género con respecto a dos dimensiones distintas de la toma individual de decisiones bajo incertidumbre: en primer lugar, el hecho de correr riesgos y, en segundo, la sensibilidad de la toma de riesgos a variaciones en la prima de riesgo. Limitamos nuestro análisis al dominio de las ganancias. Los datos que aquí analizamos fueron recogidos en un número de ocasiones en las que el test de loterías introducido por Sabater-Grande y Georgantzís (2002) fue utilizado como método de elicitación de preferencias frente al riesgo. Este test proporciona más información sobre la sensibilidad de los sujetos a incrementos en los pagos esperados como incentivo para elegir opciones más arriesgadas. Esta dimensión de las preferencias frente al riesgo la denominamos aquí sensibilidad a las primas de riesgo.

El test de loterías utilizado enfrenta a los sujetos a decisiones dentro de un amplio rango de probabilidades. Esta es una de las razones por las que nuestra metodología no posee los defectos identificados por Fehr-Duda y otros (2006), relativos a la dependencia de las diferencias de género a rangos específicos de probabilidades. Esto es debido a que, en cada decisión, los sujetos afrontan alternativas correspondientes a todo el rango de probabilidades. Además, a lo largo de los paneles, los individuos son inducidos a pasar de opciones más seguras a otras más arriesgadas mediante cambios en el parámetro de retorno al riesgo. Pese a que los datos han sido obtenidos en entornos esencialmente distintos a los estudiados por Fehr-Duda y otros (2006), nuestros resultados confirman dos de las conclusiones principales obtenidas en dicho análisis: en primer lugar, las mujeres tienen una mayor aversión al riesgo que los hombres y, en segundo lugar, sienten una menor atracción por los aumentos en el parámetro de retorno al riesgo. Ello es compatible con el trabajo de Fehr-Duda y otros (2006), en el que se indicaba que las mujeres tienden a infravalorar las probabilidades bajas más que los hombres. 
Las diferencias de género en relación al grado de aversión al riesgo han sido observadas en análisis previos ${ }^{1}$. De hecho, que las mujeres son más aversas al riesgo que los hombres es algo aceptado de forma bastante general. Esto fue confirmado, por ejemplo, por Jianakoplos y Bernasek (1998) y Powel y Ansic (1997). En concreto, Powell y Ansic (1997) demuestran que las mujeres corren menos riesgos que los hombres en tareas de laboratorio. Sin embargo, Schubert y otros (1999), Powel y Ansic (1999) y Cadsby y Maynes (2005) argumentan que esta conclusión es específica a determinados contextos, mientras que no es cierta en algunos otros. De hecho, Schubert y otros (1999) encuentran que las mujeres son, en promedio, más aversas al riesgo que los hombres en apuestas abstractas en el dominio de las ganancias, menos aversas al riesgo en el de las pérdidas, y no muy diferentes de los hombres en ambos dominios si nos encontramos en contextos de riqueza. Concluyen que el comportamiento ante el riesgo observado en los datos tomados en cuestionarios previos puede ser debido a las diferencias de oportunidades entre hombres y mujeres, más que a estereotipos en las actitudes frente al riesgo. Más recientemente, Harris y otros (2006) especifican cuatro dominios distintos en los que estudiar las diferencias de género: apuestas, salud, ocio y toma de riesgos social. Concluyen que, si bien el género no es significativo en la propensión a correr riesgos a nivel social, existen diferencias de género en los otros tres dominios.

Contrariamente a lo que indican estos moderados pero conclusivos resultados en relación con el mayor grado de aversión al riesgo de las mujeres, psicólogos como Meier-Pesti (2005) fueron más allá, observando que la aversión al riesgo está positivamente correlacionada con la feminidad ${ }^{2}$. Por ello, dado que las mujeres poseen rasgos de masculinidad y viceversa, los estudios existentes basados en el sexo biológico pueden infravalorar el efecto mencionado del factor femenino en la toma individual de decisiones bajo incertidumbre.

En cuanto a la aversión al riesgo, encontramos que las mujeres son más aversas al riesgo que los hombres. Con respecto a la sensibilidad a las primas de riesgo, obtenemos que las mujeres se sienten menos atraídas que los hombres a las primas lineales al riesgo utilizadas en el diseño de los cuatro paneles.

El artículo está organizado de la siguiente forma. En la sección 2 se presentan los detalles sobre el test de los paneles de loterías. En la sección 3 describimos el diseño experimental. En la sección 4 se discuten los resultados. Por último, en la sección 5 se muestran las conclusiones.

\footnotetext{
${ }^{1}$ ECKEL Y GROSSMAN (2008) revisan estos resultados a partir de medidas experimentales de riesgo, buscando evidencia de diferencias sistemáticas en el comportamiento de hombres y mujeres.

${ }^{2}$ La feminidad medida con tests de personalidad específicos.
} 


\section{El test de paneles de loterías}

Sea una lotería $(q, X)$ que implica una probabilidad $q$ de ganar $X$ (nada, en caso contrario). Consideremos un continuo de loterías de este tipo que compensan opciones más arriesgadas con un incremento en el pago esperado. Formalmente, cada continuo de loterías lo denotaremos con el par $(c, r)$, que corresponde, respectivamente, al pago seguro de $c$ por encima del cual el pago esperado se incrementa $r$ veces por la probabilidad de no ganar nada. Por tanto,

$$
q \cdot X(q)=c+(1-q) \cdot r \Rightarrow X(q)=\frac{c+(1-q) \cdot r}{q}
$$

Con tal de simplificar la decisión que deben tomar los sujetos, hemos utilizado varios paneles de loterías, y cada uno de ellos corresponde a una versión discreta de los continuos de loterías descritos anteriormente. Se les pide a los sujetos que escojan la lotería que prefieren en cada uno de los cuatro paneles. La Tabla 1 presenta el pago correspondiente al resultado favorable de cada lotería cuya probabilidad $(q)$ aparece en la parte superior de cada columna. Estos paneles se han generado utilizando $c=1$ y $r=0,1 ; 1 ; 5 ; 10$ para los paneles 1 a 4 respectivamente.

TABLA 1

\section{LOS CUATRO PANELES DE LOTERÍAS}

\begin{tabular}{|c|c|c|c|c|c|c|c|c|c|c|}
\hline \multicolumn{2}{|c|}{ Panel 1} & \multirow[b]{2}{*}{0,9} & \multirow[b]{2}{*}{0,8} & \multirow[b]{2}{*}{0,7} & \multirow[b]{2}{*}{0,6} & \multirow[b]{2}{*}{0,5} & \multirow[b]{2}{*}{0,4} & \multirow[b]{2}{*}{0,3} & \multirow[b]{2}{*}{0,2} & \multirow[b]{2}{*}{0,1} \\
\hline$q$ & 1 & & & & & & & & & \\
\hline$X €$ & 1 & 1,12 & 1,27 & 1,47 & 1,73 & 2,10 & 2,65 & 3,56 & 5,40 & 10,90 \\
\hline \multicolumn{11}{|l|}{ Elección } \\
\hline \multicolumn{11}{|c|}{ Panel 2} \\
\hline$q$ & 1 & 0,9 & 0,8 & 0,7 & 0,6 & 0,5 & 0,4 & 0,3 & 0,2 & 0,1 \\
\hline$X €$ & 1 & 1,20 & 1,50 & 1,90 & 2,30 & 3,00 & 4,00 & 5,70 & 9,00 & 19 \\
\hline \multicolumn{11}{|l|}{ Elección } \\
\hline \multicolumn{11}{|c|}{ Panel 3} \\
\hline$q$ & 1 & 0,9 & 0,8 & 0,7 & 0,6 & 0,5 & 0,4 & 0,3 & 0,2 & 0,1 \\
\hline$X €$ & 1 & 1,66 & 2,50 & 3,57 & 5,00 & 7,00 & 10,00 & 15,00 & 25 & 55 \\
\hline \multicolumn{11}{|l|}{ Elección } \\
\hline \multicolumn{11}{|c|}{ Panel 4} \\
\hline$q$ & 1 & 0,9 & 0,8 & 0,7 & 0,6 & 0,5 & 0,4 & 0,3 & 0,2 & 0,1 \\
\hline$X €$ & 1 & 2,20 & 3,80 & 5,70 & 8,30 & 12,00 & 17,50 & 26,70 & 45 & 100 \\
\hline Elección & & & & & & & & & & \\
\hline
\end{tabular}




\subsection{Entorno teórico y predicciones}

Supongamos que la recompensa $X$ (obtenida con probabilidad $q$ ) está diseñada para compensar el riesgo que implica la probabilidad $1-q$ del resultado desfavorable $(X=0)$, tal como implica la expresión (1). Asumamos que un individuo escoge la lotería con $q_{R} \in(0,1)$ en el panel que ofrece un premio $R<r$. Esto es, nos centramos aquí en soluciones interiores y discutiremos más adelante los casos extremos.

Resulta fácil comprobar que un sujeto con utilidad $U(X)=X^{1 / t}$, a quien se le ofrece un panel continuo ${ }^{3}$ de todas las posibles loterías diseñadas con el esquema de primas de riesgo lineales descritos en la ecuación (1), maximizaría su utilidad esperada escogiendo la lotería $\left(q^{0}, X\left(q^{0}\right)\right)$, donde

$$
q^{0}=\left(1-\frac{1}{t}\right) \cdot\left(1+\frac{c}{r}\right)
$$

También es fácil comprobar que las condiciones de segundo orden para un máximo se satisfacen ${ }^{4}$ siempre y cuando $t>1$. Esto refleja el hecho de que los sujetos neutrales al riesgo $(t=1)$ y los amantes del riesgo $(t<1)$ escogerían la opción más arriesgada posible ${ }^{5}$, puesto que su utilidad esperada, por el propio diseño de la prima, es estrictamente decreciente en $q$.

De la expresión (2), se puede comprobar que $\partial q^{0} / \partial r<0$, lo que implica un comportamiento consistente a lo largo de los diferentes escenarios en los que distintos premios suponen la elección de menores $q$ para $r$ mayores (dada la restricción obvia $\left.q^{0} \leq 1\right)$. Esto es cierto siempre y cuando la $t$ de un sujeto no sea demasiado alta, de forma que el resultado $c$ sea el preferido para cualquiera de las loterías disponibles en el panel. En tal caso, un sujeto podría escoger $q=1$ en paneles con distintas primas de riesgo. De hecho, la condición necesaria para preferir alguna opción arriesgada frente al pago seguro es que $r>c \cdot(t-1)$.

\section{Diseño experimental}

Los sujetos eligen en una tarea basada en los paneles de loterías de Sabater-Grande y Georgantzís (2002). Cada panel de loterías corresponde a una versión discreta

\footnotetext{
${ }^{3}$ Ignoramos, por el momento, las opciones discretas de que disponen los sujetos en las sesiones experimentales.

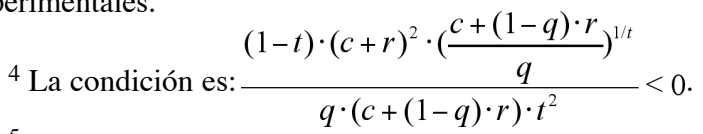

${ }^{5}$ La hipótesis de Utilidad Esperada ha sido criticada habitualmente por su explicación de la neutralidad al riesgo, la aversión al riesgo y el amor al riesgo por la forma de la función de utilidad. Sin embargo, dado que nuestro entorno de trabajo es compatible con esta conocida forma de verlo, mantenemos la terminología según la cual las actitudes frente al riesgo son debidas a la forma de la utilidad funcional con respecto a las recompensas.
} 
de un continuo de loterías como el descrito anteriormente. Cada sujeto debe escoger la opción preferida de cada panel.

Obsérvese que cuanto más a la derecha escoge un sujeto, menos averso al riesgo es, mientras que los sujetos amantes del riesgo o neutrales ante el riesgo siempre escogerán $q=0,1$ en todos los paneles. Los paneles están diseñados de tal modo que el riesgo se compensa por un incremento lineal, diferente para cada panel, en el pago monetario esperado. Los datos obtenidos de esta elección de loterías se utilizan para construir un índice del grado de aversión al riesgo de los sujetos definido como el promedio de las elecciones en los distintos paneles. Así, la elección de una probabilidad media más alta implica un mayor grado de aversión al riesgo. Sabater-Grande y Georgantzís (2002) analizan otras propiedades del test y su interpretación en términos de teorías de toma de decisiones arriesgadas.

Todas las sesiones se llevaron a cabo en el Laboratori d'Economia Experimental (LEE) de la Universitat Jaume I (Castellón). Los sujetos fueron estudiantes de temas relacionados con la economía, y cada uno participó en una sola sesión. Nuestra base de datos consiste en 668 decisiones individuales respecto a los cuatro paneles presentados en la Tabla 1.

El conjunto de datos incluye observaciones tomadas en diferentes entornos de incentivos. Pese a que la mayoría de observaciones provienen de escenarios hipotéticos, 36 de ellas corresponden a comportamiento en situaciones con recompensa monetaria real, y 31 corresponden a sesiones cuya recompensa se tradujo en notas de clase. Para los grupos compensados con dinero y notas de clase, se utilizó el mismo tipo de cambio y el mismo método de pago. Concretamente, se informó a los sujetos de que uno de los cuatro paneles sería escogido aleatoriamente para la recompensa en caso de obtener un resultado favorable. De este modo, la elección del sujeto en el panel escogido aleatoriamente se utilizaría para efectuar la lotería que determinaría si el sujeto gana la cantidad correspondiente al resultado favorable o nada, excepto en el caso de que el sujeto hubiera escogido el pago seguro $(q=1)$ para ese panel específico, caso en el que la lotería no debería realizarse.

Dado que nuestro principal objetivo era utilizar el test como un experimento preliminar sobre aversión al riesgo, el contexto hipotético es un modo más barato y rápido para elicitar preferencias al riesgo, pues no requiere realizar las loterías y no crea efectos de dotación incontrolados. De cualquier modo, aplicamos el test MannWhitney y comprobamos que, efectivamente, no existen diferencias significativas entre los comportamientos declarados y revelados de nuestros sujetos.

\section{Resultados}

Presentamos en esta sección los resultados en un modo que facilita la comparación entre las poblaciones de sujetos hombre y sujetos mujer.

En primer lugar, la figura 1 muestra las frecuencias relativas de las elecciones medias en los cuatro paneles. En comparación con las mujeres, las elecciones de los 
hombres son significativamente más dispersas en el espacio de probabilidad. De hecho, las elecciones de las mujeres están, en media, más cerca de la probabilidad central $q=0,5$.

En la figura 2 se presenta una visión más detallada de las elecciones de los sujetos en cada panel. La figura incluye histogramas de las elecciones por panel y por género.

\section{FIGURA 1}

\section{HISTOGRAMA DE ELECCIONES MEDIAS EN LOS CUATRO PANELES, POR GÉNERO}

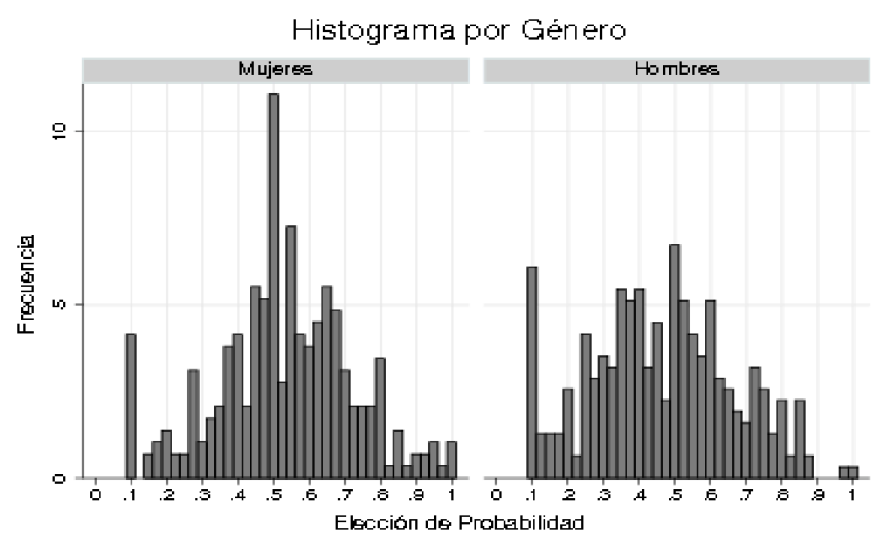

FIGURA 2

HISTOGRAMAS DE LAS ELECCIONES POR PANEL Y POR GÉNERO

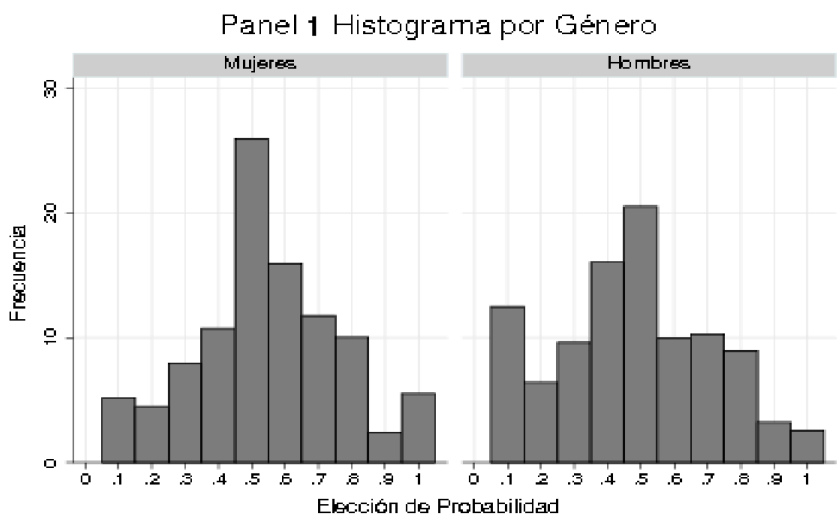

a) Panel 1 


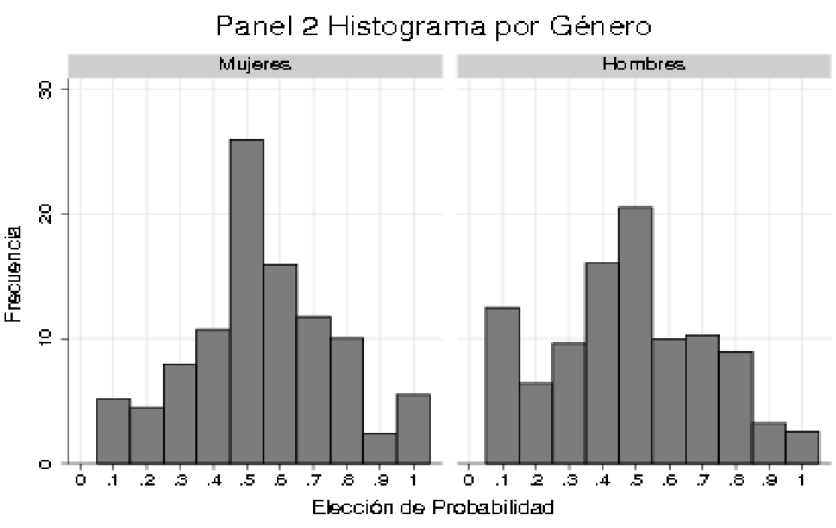

b) Panel 2

Panel 3 Histograma por Género

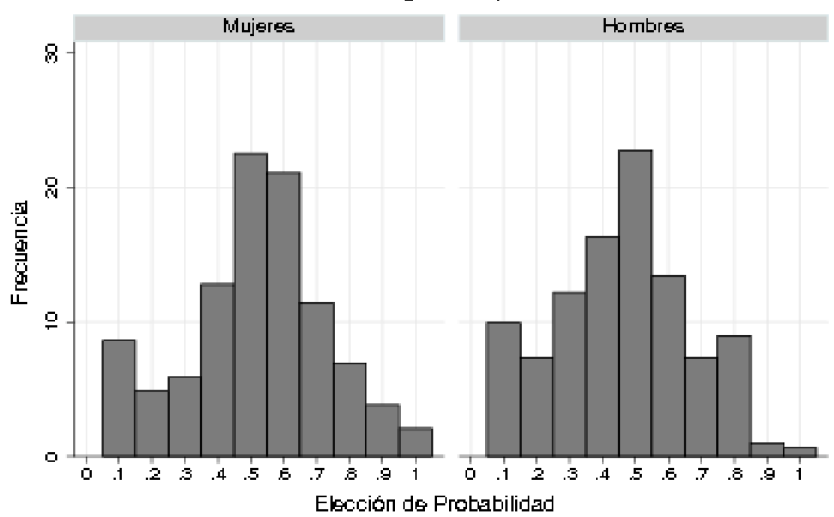

c) Panel 3

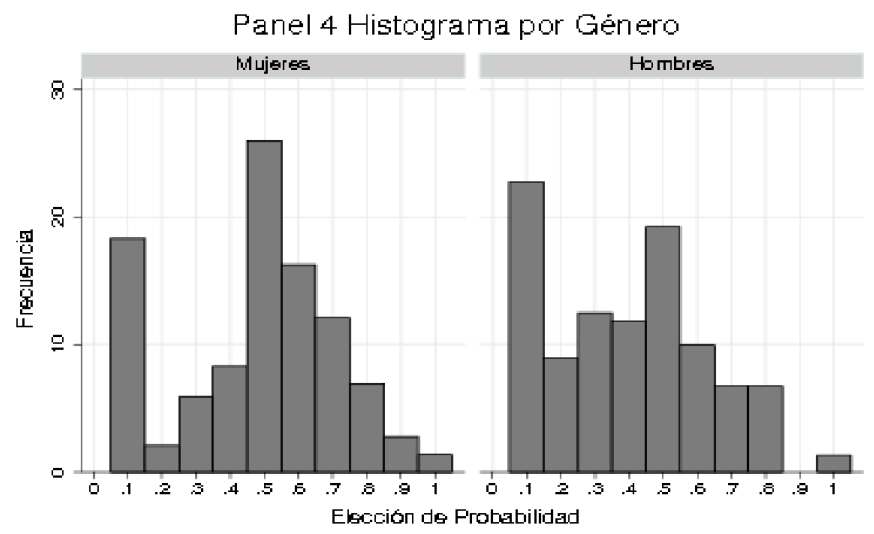

d) Panel 4 
Obsérvese que, en general, los resultados anteriores extraídos de la figura 1 se reproducen aquí. Además, cabe destacar que, en comparación con los hombres, las mujeres escogen más frecuentemente la opción segura en los cuatro paneles.

Dichos resultados también pueden notarse observando los estadísticos descriptivos presentados en la Tabla 2. Una conclusión clara es que las mujeres escogen opciones menos arriesgadas que los hombres. Y esto es cierto para los cuatro paneles. Puede confirmarse con el hecho de que, en cada panel, las mujeres escogen la opción segura $q=1$ con más frecuencia que los hombres.

TABLA 2

ESTADÍSTICOS DESCRIPTIVOS, POR GÉNERO

\begin{tabular}{|c|c|c|c|c|}
\hline & Panel 1 & Panel 2 & Panel 3 & Panel 4 \\
\hline \multicolumn{5}{|c|}{ Mujeres } \\
\hline$N$ & 289 & 289 & 289 & 289 \\
\hline Mediana & 0,50 & 0,50 & 0,50 & 0,50 \\
\hline Media & 0,58 & 0,55 & 0,52 & 0,50 \\
\hline Desv. típica & 0,27 & 0,22 & 0,21 & 0,23 \\
\hline \multicolumn{5}{|c|}{ Hombres } \\
\hline$N$ & 312 & 312 & 312 & 312 \\
\hline Mediana & 0,50 & 0,50 & 0,50 & 0,40 \\
\hline Media & 0,52 & 0,49 & 0,46 & 0,39 \\
\hline Desv. típica & 0,29 & 0,24 & 0,21 & 0,23 \\
\hline
\end{tabular}

La significatividad en las diferencias entre las elecciones de hombres y mujeres se confirma con los resultados de los tests presentados en la Tabla 3. En la tabla también se muestran los tests de las diferencias entre las elecciones, entre los distintos paneles, entre hombres y mujeres. Se observa que las elecciones de los hombres no varían sustancialmente entre los paneles 2 y 3 . 
TABLA 3

TESTS NO PARAMÉTRICOS PARA LA OBSERVACIÓN DE DIFERENCIAS ENTRE ELECCIONES DE HOMBRES Y MUJERES

\begin{tabular}{|c|c|c|c|c|c|c|c|c|}
\hline & \multicolumn{7}{|c|}{ Mujeres } \\
\hline & & Panel 1 & & Panel 2 & & Panel 3 & & Panel 4 \\
\hline \multirow{14}{*}{$\begin{array}{c}\mathbf{H} \\
\mathbf{o} \\
\mathbf{m} \\
\mathbf{b} \\
\mathbf{r} \\
\mathbf{e} \\
\mathrm{s}\end{array}$} & Panel 1 & 2,828 & 2,281 & & 2,576 & & 2,996 & \\
\hline & & $(\mathbf{0 , 0 0 5 )}$ & $(0,022)$ & & $(0,010)$ & & $(0,003)$ & \\
\hline & & 2,605 & & & & & & \\
\hline & & $(0,010)$ & & & & & & \\
\hline & Panel 2 & - & & 3,610 & & - & & - \\
\hline & & & & $(\mathbf{0 , 0 0 0 )}$ & & & & \\
\hline & & 0,950 & & & & & & \\
\hline & & $(0,342)$ & & & & & & \\
\hline & Panel 3 & - & & - & & 3,506 & & - \\
\hline & & & & & & $(\mathbf{0 , 0 0 0 )}$ & & \\
\hline & & 6,955 & & & & & & \\
\hline & & $(0,000)$ & & & & & & \\
\hline & Panel 4 & - & & - & & - & & 4,552 \\
\hline & & & & & & & & $(0,000)$ \\
\hline
\end{tabular}

Los caracteres en negrita corresponden a los resultados de los tests Mann-Whitney. El resto, corresponde al test de rangos de Wilcoxon.

Completamos con la figura 3 el cuadro de nuestros resultados. En esta figura se muestran las distribuciones acumuladas de las elecciones por panel. En los cuatro paneles, la distribución de las elecciones de las mujeres se encuentra por debajo de la de los hombres. Es decir, las mujeres muestran más aversión al riesgo que los hombres. Las distribuciones son significativamente diferentes entre ambos géneros, lo que se confirma con un test de Kolmogorov-Smirnov. 
FIGURA 3

\section{DISTRIBUCIONES ACUMULADAS POR GÉNERO}
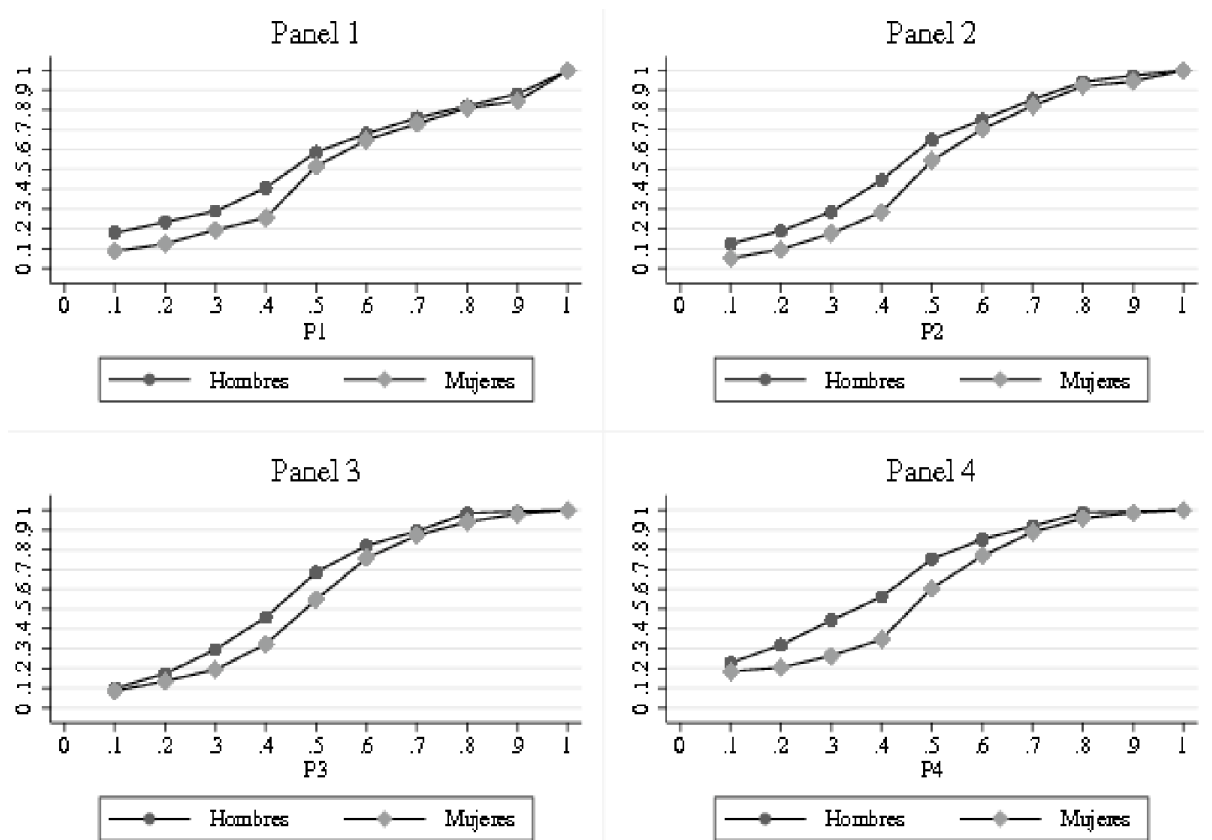

Finalmente, la Tabla 4 ofrece los resultados de un modelo de probabilidad de elección diseñado para testar si hay diferencias estructurales en las elecciones debidas al género. El modelo estimado se puede escribir como $q_{i j}=C+\beta \cdot r_{j}+e_{i}$, donde $q_{i j}$ es la elección del individuo $i$ en el panel $j, C$ es una constante y $r_{j}$ es la prima con la que se aumentan las ganancias esperadas para compensar decisiones más arriesgadas en el panel $j$. 
TABLA 4

MODELO DE PROBABILIDAD DE ELECCIÓN

\begin{tabular}{|c|c|c|c|c|}
\hline Prob. elegida & Coeficiente & Estadístico t & & \\
\hline Hombre & 0,506 & $0,010 * * *$ & & \\
\hline$r_{\text {hombre }}$ & $-0,011$ & $0,002 * * *$ & & \\
\hline Mujer & 0,567 & $0,010 * * *$ & & \\
\hline$r_{\text {mujer }}$ & $-0,009$ & $0,002 * * *$ & & \\
\hline$R^{2}$ & \multicolumn{2}{|c|}{0,813} & & \\
\hline$\hat{\sigma}$ & \multicolumn{2}{|c|}{0,812} & & \\
\hline$F(4,2.400)$ & \multicolumn{2}{|c|}{$2.604,16^{* * *}$} & & \\
\hline \multirow{4}{*}{ Test de Chow } & & $\alpha_{1}($ hombre $)$ & $=$ & $\alpha_{2}($ mujer $)$ \\
\hline & & $\beta_{l}\left(r_{\text {hombre }}\right)$ & $=$ & $\beta_{2}\left(r_{\text {mujer }}\right)$ \\
\hline & & $F(2,2.400)$ & $=$ & 24,47 \\
\hline & & Prob $>F$ & $=$ & 0,000 \\
\hline
\end{tabular}

La estimación indica que:

Resultado 1. Las mujeres son más aversas al riesgo que los hombres.

Resultado 2. Las mujeres son menos sensibles que los hombres a las primas de riesgo.

La significatividad del estimador de la constante para cada grupo (Hombre, Mujer) nos lleva al primer resultado. Al segundo resultado llegamos al observar la significatividad de los estimadores de las pendientes $\left(r_{\text {hombre }}, r_{\text {mujer }}\right)$. Ambos resultados se confirman mediante la significatividad global del modelo, tal como indica el test de Chow $(\mathrm{p}=0,000)$. Por lo tanto, podemos afirmar que hay un cambio estructural entre grupos en el modelo estimado.

\section{Conclusiones}

Hemos analizado las diferencias de género con respecto a dos dimensiones de la toma individual de decisiones bajo incertidumbre: en primer lugar, el hecho de correr riesgos y, en segundo, la sensibilidad del correr riesgos a variaciones en las primas de riesgo. Presentamos los resultados obtenidos en la tarea de elección de loterías introducida por Sabater-Grande y Georgantzís (2002), diseñada para medir el grado de aversión al riesgo de los sujetos y su sensibilidad a las primas de riesgo. Hemos delimitado el estudio de toma de decisiones al dominio de las ganancias. 
Con respecto a la aversión al riesgo, obtenemos que las mujeres son más aversas al riesgo que los hombres. Esto se confirma en el contexto de los cuatro paneles, en los que el riesgo está más que compensado por aumentos lineales en los pagos esperados a medida que decrece la probabilidad de ganar.

Analizando la sensibilidad a las primas, se obtiene que las mujeres se sienten menos atraídas que los hombres por los aumentos lineales de primas de riesgo utilizados en el diseño de los cuatro paneles. Por tanto, en comparación con los hombres, las decisiones de las mujeres tienden a una mayor aglomeración cerca de la opción central $q=0,5$. Para identificar esta pauta en otros dominios económicos como, por ejemplo, el comportamiento en mercados financieros, decisiones en pólizas de seguros, etc., se hace necesaria más investigación en este campo.

\section{Referencias}

[1] CADSBY, C.B. y MAYNES, E. (2005). «Gender, risk aversion, and the drawing power of equilibrium in an experimental corporate takeover game», Journal of Economic Behavior y Organization 56, pp. 36-59.

[2] ECKEL, C.C. y GROSSMAN, P.J. (2008). «Men, women and risk aversion: Experimental evidence», en C. Plott y V. Smith (eds.), Handbook of Experimental Economics Results, vol. 1, cap. 113, pp. 1061-1073, Elsevier.

[3] FEHR-DUDA, H., GENNARO, M. y SCHUBERT, R. (2006). «Gender, financial risk and probability weights», Theory y Decision 60, pp. 283-313.

[4] HARBAUGH, W., KRAUSE, K. y VESTERLUND, L. (2002). «Risk attitudes of children and adults: Choices over small and large probability gains y losses», Experimental Economics 5, pp. 53-84.

[5] HARRIS, C.R., JENKINS, M. y GLASER, D. (2006). «Gender differences in risk assessment: Why do women take fewer risks than men?», Judgment y Decision Making 1, pp. 48-63.

[6] JIANAKOPLOS, N. y BERNASEK, A. (1998). «Are women more risk averse?», Economic Inquiry 36 , pp. 620-630.

[7] MEIER-PESTI, K. (2005). «Effects of masculinity and feminity on financial risktaking: Some evidence from an experimental study on gender salience», XXX encuentro de la International Association of Research in Economic Psychology, Praga.

[8] POWELL, M. y ANSIC, D. (1997). «Gender differences in risk behavior in financial decision making: An experimental analysis», Journal of Economic Psychology 18 (6), pp. 605-628.

[9] POWELL, M. y ANSIC, D. (1999). «Gender differences in financial decision-making: a new approach for experimental economic analysis», artículo no publicado (presentado en la AEA Conference, Nueva York, enero de 1999).

[10] SABATER-GRANDE, G. y GEORGANTZÍS, N. (2002). «Accounting for risk aversion in repeated prisoners' dilemma games: An experimental test», Journal of Economic Behavior y Organization 48, pp. 37-51.

[11] SCHUBERT, R., BROWN, M., GYSLER, M. y BRACHINGER, H. (1999). «Financial decision making: are women really more risk averse?», American Economic Review (Papers y Proceedings) 89, pp. 381-385. 